\title{
Syncope in the older patient: initial evaluation and emergency department management according to 2018 European Society of Cardiology guidelines
}

\author{
Andrea Ungar, Martina Rafanelli \\ Syncope Unit, Department of Geriatrics, Careggi University Hospital, Florence, Italy
}

\begin{abstract}
The prevalence of syncope increases with advancing age and is associated with significant morbidity and mortality. The diagnosis within this population can be complex due to atypical presentations, amnesia for events, absence of witnesses and the overlap with other clinical presentations, such as falls. The recently updated European Society of Cardiology guidelines on syncope propose structured assessment and management, which is also applicable to the older patient, with special attention to some additional features, pertinent to age-related comorbidity and frailty.
\end{abstract}

\section{Introduction}

Older patients frequently experience syncope. The diagnostic and therapeutic management may be complex in this group of age, particularly in the presence of other comorbidities or cognitive impairment. Morbidity related to syncope is more common in the elderly and ranges from loss of confidence, depressive illness and fear of falling, to fractures and consequent institutionalization. Moreover, advanced age is associated with short and long-term morbidity and mortality after syncope. ${ }^{1}$ A standardized approach may obtain a definite diagnosis in more than $90 \%$ of the cases, may

Correspondence: Andrea Ungar, Syncope Unit, Department of Geriatrics, Careggi University Hospital, Viale Pieraccini 6, 50139 Florence, Italy.

Tel.: +39.055.2758126.

E-mail: aungar@unifi.it

Key words: Syncope; Older patient; Initial evaluation; Emergency department; Guidelines.

Contributions: the authors contributed equally.

Conflict of interest: the authors declare no potential conflict of interest.

Funding: none.

Received for publication: 7 May 2018.

Revision received: 7 June 2018.

Accepted for publication: 12 June 2018.

This work is licensed under a Creative Commons Attribution 4.0 License (by-nc 4.0).

(C) Copyright A Ungar and M. Rafanelli, 2018

Licensee PAGEPress, Italy

Emergency Care Journal 2018; 14:7543

doi:10.4081/ecj.2018.7543 reduce diagnostic tests and rate of hospitalization. ${ }^{2}$

The present article will review the characteristics of syncope in the elderly and will focus on initial evaluation and Emergency Department (ED) management, as proposed in the 2018 version of the European Society of Cardiology (ESC) guidelines on syncope. ${ }^{1}$

Syncope is a transient loss of consciousness (TLOC) due to a transient global cerebral hypo-perfusion, characterized by a rapid onset, short duration, spontaneous and complete recovery. ${ }^{1}$

TLOC is defined as a state of real or apparent LOC with loss of awareness, characterized by amnesia for the period of unconsciousness, abnormal motor control, loss of responsiveness, and a short duration. ${ }^{1}$ There are two main groups of TLOC, those due to head trauma and non-traumatic TLOC. ${ }^{1}$ A state that resembles prodromal symptoms of syncope, but which is not followed by LOC, is defined as pre-syncope. ${ }^{1}$

\section{Epidemiology}

Syncope is frequent in the general population ${ }^{3}$ and is responsible for ED attendances and hospital admissions in 3\% and 1\% of the cases, respectively. ${ }^{2}$ In the latest report of the Framingham Offspring study, $10 \%$ of the 7814 participants (mean age 51, range 20-96 years) reported at least one episode of syncope during a 17years follow up. ${ }^{3}$ The incidence rate of the first syncope was 6.2 per 1000 person years, with a sharp increase after 70 years from 5.7 events per 1000 person years in men aged 60 to 69 to 11.1 in men aged 70 to 79 - equivalent to an estimated 10 year cumulative incidence of $6 \% .^{3-6}$

\section{Classification of syncope}

Reflex syncope is the most frequent cause at all ages, ${ }^{7}$ while orthostatic hypotension $(\mathrm{OH})$ is a frequent cause of syncope in very old patients. ${ }^{2}$ In the elderly, multiple causes are often present and the diagnosis within this population can be complex due to atypical presentations, amnesia for events, absence of witnesses and overlap with other clinical presentations, such as unexplained falls. ${ }^{8}$ Therefore, an early and detailed assessment, through a standardized guideline-based approach, is mandatory. The principal causes of syncope are listed in Brignole et al. ${ }^{1}$

\section{Reflex (neurally-mediated) syncope}

\section{Vasovagal syncope}

Vasovagal Syncope (VVS) is induced by triggers such as fear, pain and instrumentation or induced by orthostatic stress or a hot environment. In older patients, the presentation is often atypical. Syncope can occur with uncertain stimuli or even apparently with- 
out triggers. Prodromal symptoms may be absent or short, and loss of consciousness may start abruptly, leading to collapse and injuries. ${ }^{7}$ Nausea, blurred vision and diaphoresis are mostly common in VVS, whereas dyspnea is more predictive of cardiac syncope.$^{8}$ During the syncopal phase, myoclonic movements are rare in older subjects, probably because of a lack of asystolic response and a slower reduction in Systolic Blood Pressure (SBP). ${ }^{9}$

A VVS which occurs upon standing may result in collapse, which can be misidentified as a fall, thereby rendering the clinical findings of these two conditions, very similar. In this context, retrograde amnesia was demonstrated in patients with syncope induced in a syncope clinic; indeed about $25 \%$ of patients fail to recall their prodromal symptoms and TLOC during tilt-induced syncope. ${ }^{10}$

\section{Carotid sinus syncope}

Carotid sinus hypersensitivity (CSH) can manifest as cardioinhibitory (CI-CSH) (asystole $\geq 3$ seconds during Carotid Sinus Massage, CSM) or vasodepressor (a fall in SBP $\geq 50 \mathrm{mmHg}$ during $\mathrm{CSM}$ ) or mixed $\mathrm{CSH}$ and represents a positive response to CSM in an asymptomatic patient. ${ }^{1} \mathrm{CSH}$ could indicate an abnormal reflex, which may have a role in predisposing to unexplained falls. In this situation, syncope has to be considered, even if the typical sequence of a syncopal event cannot be recalled by the patient due to the presence of retrograde amnesia. Maggi et al. ${ }^{11}$ showed that CI-CSH in patients with a clinical diagnosis of suspected neurallymediated syncope is related to a long asystolic reflex detected by an Implantable Loop Recorder at the time of the spontaneous syncope. When CSH occurs in a patient who has previously had syncope with reproduction of symptoms, this is defined as Carotid Sinus Syndrome (CSS). ${ }^{1}$

The prevalence of CSS has been estimated to range from $<4 \%$ in patients $<40$ years to $41 \%$ in those $>80$ years attending a specialized syncope facility. The related syncope has often little or no prodrome, with an increased risk of traumatic fall. Syncope recurrence is common and is reported to be $50 \%$ in 2 years. ${ }^{12}$

The ESC guidelines on pacing ${ }^{13}$ propose a 6 second cut- off for CSM-induced asystole, rather than the historical 3-second cut-off value, as the longer pause is more likely to be clinically relevant.

Moreover, several studies have demonstrated that isolated cardio-inhibitory response does not exist, since vasodepression is present in nearly all the patients, with variable intensity. ${ }^{14}$ Therefore, a revision of conventional CSS classification has been proposed, considering CSS as a continuum between cardio-inhibition and vasodepression and including predominant cardio-inhibitory, predominant vasodepressor and mixed CSS. ${ }^{15}$ This new interpretation can influence patient's referral for treatment, since cardiac pacing is less effective in the presence of a significant vasodepressor response, which may be responsible for syncopal recurrence.

\section{Orthostatic hypotension}

$\mathrm{OH}$ is defined as a fall in SBP from a baseline value $\geq 20$ $\mathrm{mmHg}$ or diastolic $\mathrm{BP} \geq 10 \mathrm{mmHg}$ or a sustained decrease in systolic BP to an absolute value of $<90 \mathrm{mmHg}$ within 3 minutes of orthostatic position. ${ }^{1}$ Since the magnitude of blood pressure drop also depends on baseline values, a drop of $30 \mathrm{mmHg}$ might be a more appropriate criterion for $\mathrm{OH}$ in patients with supine hypertension. ${ }^{15}$

$\mathrm{OH}$ increases with age, reaching $24.3 \%$ in the 8 th decade and $30.9 \%$ in the 9 th decade, ${ }^{16}$ and has a prevalence of $12.4 \%$ in patients older than 65 years old consecutively referred to the ED

\section{for a TLOC. ${ }^{17}$}

The circulatory autonomic causes of orthostatic intolerance include initial orthostatic hypotension ( $\mathrm{IOH})$, classical orthostatic hypotension $(\mathrm{COH})$, and delayed orthostatic hypotension $(\mathrm{DOH}){ }^{1}$

$\mathrm{IOH}$, which occurs within 15 seconds of standing, may have implications in older adults, particularly when on cardiovascular medications, ${ }^{18} 15 \%$ of long-term care residents indeed fall after rising to standing ${ }^{19}$ and initial $\mathrm{OH}$ could potentially exacerbate this falling risk.

$\mathrm{DOH}$ is common in the elderly, due to impairment of compensatory reflexes and stiffer hearts, sensitive to a decrease in preload..$^{20}$ It may also represent a mild form of $\mathrm{COH}$, especially if associated with Parkinsonism or diabetes. ${ }^{21}$

Pharmacotherapy is the primary cause of $\mathrm{OH}$ in the older patient. A drug regimen based on alpha-receptor blockers, nitrates or benzodiazepines, was found to be a predictor of $\mathrm{OH}$ in this age group. ${ }^{17}$

\section{Cardiac syncope}

Cardiac causes of syncope are highly represented in the older population. ${ }^{22}$ Short-lived syncope of abrupt onset and recovery, supine, during (rather than after) exercise or associated with palpitations or chest pain, should be considered cardiac until proven otherwise. A history of heart disease is an independent predictor of cardiac syncope with a sensitivity of $95 \%$ and specificity of $45 \%{ }^{23}$ In particular, cardiac syncope should be suspected in patients with known or suspected left ventricular systolic dysfunction, valvular disease, left ventricular outflow tract obstruction, in those with an abnormal surface electrocardiogram (ECG) and where the clinical context and concomitant investigations suggest pulmonary embolism. A neurally-mediated cause of symptoms cannot be assumed in any patient with these clinical and diagnostic features until a cardiac cause has been effectively ruled-out.

Brady- and tachy-arrhythmias are the most common cardiac causes of cardiac syncope. Atrial fibrillation (AF) is the most common cardiac arrhythmia in adults with a prevalence rising from 1 to $2 \%$ in the general population to nearly $5 \%$ in community dwelling people aged over 65 years old. ${ }^{24}$ In a large cohort of community dwelling elderly patients, objectively diagnosed AF was associated with syncope, independent of stroke, cardiovascular drugs and other confounders. AF was also associated with one or more falls in the past year in those aged $65-74$ years. ${ }^{25}$ Guidelines ${ }^{1}$ and clinical scoring systems ${ }^{26,27}$ for identifying high-risk patients include arrhythmias as a predictor of death and adverse events.

\section{Multifactorial causes of syncope in older patients}

Elderly patients often have multiple coexisting potential causes of syncope, and a definite diagnosis may be difficult. In a population of 873 consecutive patients older than 65 years, the rate of complex diagnoses [more than one diagnosis on an active standing test, Tilt Testing (TT) and CSM] was $23 \%$ and the most frequent association was between $\mathrm{OH}$ and VVS on TT in $15.8 \%$ of the cases. ${ }^{16}$ In a sample of very old patients evaluated in a syncope and falls clinic, the most common causes of syncope in the elderly were $\mathrm{OH} /$ post-prandial hypotension, followed by cardiac disorders; while reflex syncope was less common..$^{28}$

It is therefore useful to complete a comprehensive evaluation, without stopping at the first diagnosis. 


\section{Overlapping between syncope and falls}

The incidence of syncope in older patients is likely to be higher than current estimates, due to the overlap with other presentations such as falls.

A fall is defined unexplained, when happens without accidental or clear medical conditions. ${ }^{29}$ Unexplained falls represent a common cause of hospital admission and are associated with increased healthcare costs. ${ }^{30}$ Especially in older adults in whom the circumstances of a fall are unclear, because of the lack of witnesses and amnesia for the episode, a misdiagnosed syncope may underlie an unexplained fall. About $20 \%$ of cardiovascular syncope in patients older than 70 years old presents as a fall, especially in patients with CSS and $\mathrm{OH}$. More than $20 \%$ of the older patients with CSS complain of falls as well as syncope. ${ }^{31}$ In patients older than 60 years admitted to the hospital because of a fall or syncope, fallers who had CSS during CSM, showed retrograde amnesia for the loss of consciousness, more frequently than patients with syncope. ${ }^{32}$ Furthermore, over one third of the falls in patients in orthopedic wards are unexplained, particularly in those with depressive symptoms and syncopal spells, ${ }^{33}$ underscoring the importance of a comprehensive clinical history and assessment at the very beginning of the medical pathway.

Those unexplained falls, initially considered as not being due to an episode of syncope, but in which a final diagnosis of syncope is confirmed after a diagnostic work-up, have been recently defined as syncopal falls. ${ }^{34}$

Falls can result from a postural drop in BP. Two to ten \% of the falls in older adults may occur secondary to impaired hemodynamic responses and loss of consciousness is estimated to result in as many as $10 \%$ of falls. ${ }^{35}$ In many cases, loss of consciousness is avoided, but increased susceptibility to falling remains through pre-syncope and associated physiological impairments. ${ }^{36}$

Impaired orthostatic BP recovery, delayed recovery or sustained $\mathrm{OH}$ are independent risk factors for future falls, unexplained falls, and injurious falls. ${ }^{37}$

\section{Initial evaluation}

The initial evaluation, which consists of clinical history, physical examination, an active standing test and a 12-lead ECG, should answer some key questions: ${ }^{1}$ i) Was the event TLOC? ii) In the case of TLOC, is it of syncopal or non-syncopal origin? iii) In suspected syncope, is there a clear aetiological diagnosis? iv) Is there evidence to suggest a high risk of cardiovascular events or death?

Brignole et al. ${ }^{1}$ outlined the steps for initial evaluation and risk stratification of patients with syncope.

The clinical history should include the collection of comorbidities, physical frailty and loco-motor disabilities, details of cognitive status, social circumstances, injuries, impact of the event on functional capacity. Eyewitness accounts should be collected, given the frequent presence of retrograde amnesia in the elderly. Particular attention should be paid to the time of the day, season, relationship with meals, micturition, body position, drugs, length of treatment and time-relationship between drug consumption and appearance of adverse effects. ${ }^{1}$

Precise details of the drug regimen have to be collected as numerous drugs, e.g., alpha-receptor blockers, nitrates or benzodiazepines, were found to be predictors of $\mathrm{OH}$. Attention should be paid to reappraisal of the drug regimen in the presence of $\mathrm{OH}$, in order to reduce the recurrence of syncope. ${ }^{16}$

A comprehensive physical examination, extended to include vision, cognitive status, gait, standing balance and evaluation of the loco-motor system including the feet, to assess risk factors for falls is suggested.

The active standing test consists of the measurement of BP in the supine position, immediately upon standing and after 1 to 3 minutes of standing. Given the age-related increase in $\mathrm{OH}$, standing BP measurements are mandatory in the elderly and should be repeated, preferably in the morning and/or promptly after the syncope, as the standing fall in BP is not always reproducible, especially when related to drugs or predisposing conditions. ${ }^{1}$

The 12-lead ECG is diagnostic, can remove the need for further cardiac evaluation and permits institution of treatment, in cases of: i) persistent sinus bradycardia $<40 \mathrm{bpm}$ in awake or repetitive sinus-atrial block or sinus pauses $>3 \mathrm{~s}$; ii) Mobitz II 2nd or 3rd degree atrio-ventricular block, alternating left and right bundle branch block; iii) ventricular tachycardia (VT) or rapid paroxysmal supra-ventricular tachycardia; iv) non $\square$ sustained episodes of polymorphic VT and long or short QT interval; v) acute ischemia with or without myocardial infarction. ${ }^{1}$

However, studies have shown that an ECG will determine the cause of syncope in only $5 \%$ of patients. ${ }^{38}$

The ESC guidelines on syncope ${ }^{1}$ propose the execution of CSM on the initial evaluation in patients aged $>40$ years. The test is performed under continuous HR and beat-to-beat BP monitoring, for 10 seconds, bilaterally, first in the supine and then in the upright position, on TT at an angle of $60^{\circ}$. The added diagnostic value of repeating CSM in the upright position has been documented. ${ }^{39}$ To assess the contribution of the vasodepressive component, CSM may be repeated after intravenous administration of 0.02 $\mathrm{mg} / \mathrm{Kg}$ of atropine, which eliminates vagally-induced asystole, thereby unmasking vasodepression. ${ }^{40}$ This quantification of the vasodepressive component is clinically relevant, because it has been shown that pacemaker therapy is less effective when the vasodepressive effect is large, compared with predominant cardioinhibition. ${ }^{41}$ CSM should be undertaken with caution in patients with previous transient ischemic attack, stroke, or known carotid stenosis $>70 \%{ }^{1}$

Additional tests may be performed: immediate ECG monitoring in suspected arrhythmic syncope; echocardiogram in known heart disease, or syncope secondary to cardiovascular cause; TT when there is suspicion of syncope due to $\mathrm{OH}$ or reflex syncope. Blood tests, e.g. haematocrit or haemoglobin when haemorrhage is suspected, oxygen saturation and blood gas analysis when hypoxia is suspected, troponin when cardiac ischaemia-related syncope is suspected, or D-dimer when pulmonary embolism is suspected, etc. ${ }^{1}$ In case of certain or highly likely diagnosis after the initial evaluation, no further evaluation is needed, and definitive treatment can be planned.

\section{Emergency department management of syncope based on risk stratification}

The ED management of TLOC of suspected syncopal nature should follow the three following main steps.

\section{Identify serious underlying causes of syncope}

The primary aim of an ED physician is to detect and treat acute 
underlying diseases, which most frequently determine short-term adverse events, rather than the syncope itself. This is particularly relevant in the older patient who often has comorbidities and more than one possible cause of syncope. The presence of a cardiovascular diagnosis, such as aortic stenosis or atrial fibrillation in this age group, may be coincidental rather than the attributable cause of events.

\section{Establish the risk of a serious outcome}

The second step is to distinguish between patients with lowand high-risk conditions; patients at low-risk should be discharged with adequate education but high-risk patients need hospital admission and urgent full investigation. While low-risk patients are more likely to have reflex syncope and an excellent prognosis, high-risk ones are more likely to have cardiac syncope and a worse prognosis. Structural heart disease and cardiac arrhythmias are major risk factors for sudden cardiac death and overall mortality in patients with syncope. ${ }^{42,43}$ Features that suggest low and high risk conditions are listed in Brignole et al. ${ }^{1}$

\section{Choose between discharge and hospital admission}

The rate of hospital admission for syncope, after the evaluation in the ED varies between 12 and $86 \%$. The main aim of ED physicians is to identify high-risk patients, needing early investigations and hospitalization, while unnecessary admission in low-risk patients can be harmful.

The implementation of organizational approaches, such as ED Observation Units and Syncope Clinics, offers safe and effective alternatives to admission in some cases. ${ }^{1}$

Several risk stratification tools have been validated, none of which are widely used in EDs, due to poor sensitivity and specificity. ${ }^{1}$ Risk stratification tools perform no better than clinician judgment at predicting short-term serious outcomes. ${ }^{43}$

\section{Conclusions}

The diagnostic protocol proposed by the ESC guidelines on syncope, ${ }^{1}$ is applicable at any age, but some additional features, pertinent to age-related comorbidity and frailty, warrant special attention. Comorbidity influences the diagnosis of syncope and management decisions, so a comprehensive, multifactorial evaluation extended to include cognitive assessment, physical performance and functional assessment, is recommended. Intervention is often multi-faceted. As the risk of syncope and falls is increased by polypharmacy, a careful revision of the drug regimen is essential. Unexplained falls should be evaluated as for unexplained syncope.

\section{References}

1. Brignole M, Moya A, de Lange FJ, et al. 2018 ESC Guidelines for the diagnosis and management of syncope. The Task Force for the diagnosis and management of syncope of the European Society of Cardiology (ESC) Developed with the special contribution of the European Heart Rhythm Association (EHRA). Eur Heart J 2018;00:1-69.

2. Ungar A, Mussi C, Del Rosso A, et al. Diagnosis and characteristics of syncope in older patients referred to geriatric departments. J Am Geriatr Soc 2006;54:1531-6.

3. Soteriades ES, Evans JC, Larson MG, et al. Incidence and prognosis of syncope. N Engl J Med 2002;347:878-85.

4. Moya A, Sutton R, Ammirati F, et al. Guidelines for the diag- nosis and management of syncope (version 2009): task force for the diagnosis and management of syncope of the European Society of Cardiology (ESC). Eur Heart J 2009;30:2631-71.

5. Brignole M, Menozzi C, Bartoletti A, et al. A new management of syncope: prospective systematic guideline-based evaluation of patient referred urgently to general hospitals. Eur Heart J 2006;27:76-82.

6. Alshekhlee A, Shen WK, Mackall J, et al. Incidence and mortality rates of syncope in the United States. Am J Med 2009;122:181-8.

7. Del Rosso A, Alboni P, Brignole M, et al. Relation of clinical presentation of syncope to the age of patients. Am J Cardiol 2005;96:1431-5.

8. Galizia G, Abete P, Mussi C, et al. Role of early symptoms in assessment of syncope in elderly people: results from the Italian group for the study of syncope in the elderly. J Am Geriatric Soc 2009:57:18-23.

9. Tan MP, Parry SW. Vasovagal syncope in the older patient. J Am Coll Cardiol 2008;51:599-606.

10. O'Dwyer C, Bennett K, Langan Y, et al. Amnesia for loss of consciousness is common in vasovagal syncope. Europace 2011;13:1040-5.

11. Maggi R, Menozzi C, Brignole M, et al. Cardioinhibitory carotid sinus hypersensitivity predicts an asystolic mechanism of spontaneous neurally mediated syncope. Europace 2007;9:563-7.

12. Sutton R. Carotid sinus syndrome: Progress in understanding and management. Global Cardiol Sci Practice 2014:18.

13. Brignole M, Auricchio A, Baron-Esquivias G, et al. ESC Guidelines on cardiac pacing and cardiac resynchronization therapy The Task Force on cardiac pacing and resynchronization therapy of the European Society of Cardiology (ESC). Developed in collaboration with the European Heart Rhythm Association (EHRA). Europace 2013;15:1070-118.

14. Menozzi C, Brignole M, Lolli G, et al. Follow-up of asystolic episodes in patients with cardioinhibitory, neurally mediated syncope and VVI pacemaker. Am J Cardiol 1993;72:1152-5.

15. Freeman R, Wieling W, Axelrod FB, et al. Consensus statement on the definition of orthostatic hypotension, neurally mediated syncope and the postural tachycardia syndrome. Clin Auton Res 2011;21:69-72.

16. Rafanelli M, Morrione A, Landi A, et al. Neuroautonomic evaluation of patients with unexplained syncope: incidence of complex neurally mediated diagnoses in the elderly. Clin Interv Aging 2014;9:333-8.

17. Mussi C, Ungar A, Salvioli G, et al. Evaluation of guidelines in Syncope Study 2 Group. Orthostatic hypotension as cause of syncope in patients older than 65 years admitted to emergency departments for transient loss of consciousness. J Gerontol A Biol Sci Med Sci 2009;64:801-6.

18. Robinovitch SN, Feldman F, Yang Y, et al. Video capture of the circumstances of falls in elderly people residing in long-term care: an observational study. Lancet 2013;381:47-54.

19. Ricci F, De Caterina R, Fedorowski A. Orthostatic hypotension: epidemiology, prognosis, and treatment. J Am Coll Cardiol 2015;66:848-60.

20. Gibbons CH, Freeman R. Clinical implications of delayed orthostatic hypotension: a 10-year follow-up study. Neurology 2015;85:1362-7.

21. Marrison VK, Fletcher A, Parry SW. The older patient with syncope: Practicalities and controversies. Int $\mathrm{J}$ Cardiol 2012;155:9-13.

22. Alboni P, Brignole M, Menozzi C, et al. Diagnostic value of 
history in patients with syncope with or without heart disease. J Am Coll Cardiol 2001;37:1921-8.

23. Del Rosso A, Ungar A, Maggi R, et al. Clinical predictors of cardiac syncope at initial evaluation in patients referred urgently to general hospital: the EGSYS score. Heart 2008;94:16206.

24. Kirchhof P, Benussi S, Kotecha D, et al. 2016 ESC Guidelines for the management of atrial fibrillation developed in collaboration with EACTS. Eur Heart J 2016:37:2893-962.

25. Jansen S, Frewen J, Finucane C, et al. AF is associated with self-reported syncope and falls in a general population cohort. Age Ageing 2015;44:598-603.

26. Colivicchi F, Ammirati F, Melina D, et al. OESIL (Osservatorio Epidemiologico sulla Sincope nel Lazio) study investigators. Development and prospective validation of a risk stratification system for patients with syncope in the emergency department: the OESIL risk score. Eur Heart J 2003;24:811-9.

27. Quinn J, McDermott D, Stiell I, et al. Prospective validation of the San Francisco syncope rule to predict patients with serious outcomes. Ann Emerg Med 2006;47:448-54.

28. de Ruiter SC, Wold JFH, Germans T, et al. Multiple causes of syncope in the elderly: diagnostic outcomes of a Dutch multidisciplinary syncope pathway. Europace 2017;0:1-6.

29. Masud T, Morris RO. Epidemiology of falls. Age Ageing 2001;30:3-7.

30. Scuffham P, Chaplin S, Legood R. Incidence and costs of unintentional falls in older people in the United Kingdom. J Epidemiol Commun Health 2003;57:740-4.

31. Carey BJ, Potter JF. Cardiovascular causes of falls. Age Ageing 2001;30:19-24.

32. Kenny RA, Richardson DA, Steen N, et al. Carotid sinus syndrome: a modifiable risk factor for non accidental falls in older adults (SAFE PACE). J Am Coll Cardiol 2001;38:1491-6.

33. Mussi C, Galizia G, Abete P, et al. Unexplained falls are frequent in patients with fall-related injury admitted to orthopaedic wards: The UFO Study (Unexplained Falls in Older Patients). Curr Gerontol Geriatr Res 2013. Available from: https://www.hindawi.com/journals/cggr/2013/928603/.

34. Alboni P, Coppola P, Stucci N, et al. Differential diagnosis between 'unexplained' fall and syncopal fall: a difficult or impossible task. J Cardiovasc Med (Hagerstown) 2015;16:829.

35. Shaw BH, Claydon VE. The relationship between orthostatic hypotension and falling in older adults. Clin Auton Res 2014;24:3-13.

36. Wieling W, Thijs RD, van Dijk N, et al. Symptoms and signs of syncope: a review of the link between physiology and clinical clues. Brain 2009;132:2630-42.

37. Finucane C, O'Connell MDL, Donoghue O, et al. Impaired orthostatic blood pressure recovery is associated with unexplained and injurious falls. J Am Geriatr Soc 2017;65:474-82.

38. Linzer M, Yang EH, Estes NA 3rd, et al. Diagnosing syncope. Part 1: value of history, physical examination, and electrocardiography. Clinical Efficacy Assessment Project of the American College of Physicians. Ann Intern Med 1997;126: 989-96.

39. Parry SW, Richardson DA, O'Shea D, et al. Diagnosis of carotid sinus hypersensitivity in older adults:carotid sinus massage in the upright position is essential. Heart 2000;83:22-3.

40. Solari D, Maggi R, Oddone D, et al. Assessment of the vasodepressor reflex in carotid sinus syndrome. Circ Arrhythm Electrophysiol 2014; 7:505-10.

41. Lopes R, Gonçalves A, Campos J, et al. The role of pacemaker in hypersensitive carotid sinus syndrome. Europace 2011;13: 572-5.

42. Priori SG, Blomstrom-Lundqvist C, Mazzanti A, et al. 2015 ESC Guidelines for the management of patients with ventricular rrhythmias and the prevention of sudden cardiac death: The Task Force for the Management of Patients with Ventricular Arrhythmias and the Prevention of Sudden Cardiac Death of the European Society of Cardiology (ESC). Endorsed by: Association for European Paediatric and Congenital Cardiology (AEPC). Eur Heart J 2015;36:2793-867.

43. Costantino G, Casazza G, Reed M, et al. Syncope risk stratification tools vs clinical judgment: an individual patient data meta-analysis. Am J Med 2014;127:1126.e13-25. 